\title{
Slepton pair production in association with a jet: NLO-QCD corrections and parton-shower effects
}

\author{
Barbara Jäger, ${ }^{a}$ Andreas von Manteuffel ${ }^{b}$ and Stephan Thier ${ }^{b, c}$ \\ ${ }^{a}$ Institute for Theoretical Physics, University of Tübingen, \\ Auf der Morgenstelle 14, 72076 Tübingen, Germany \\ ${ }^{b}$ PRISMA Cluster of Excellence, Institute of Physics, Johannes Gutenberg University, \\ Staudingerweg 7, 55099 Mainz, Germany \\ ${ }^{c}$ II. Institute for Theoretical Physics, Hamburg University, \\ Luruper Chaussee 149, 22761 Hamburg, Germany \\ E-mail: jaeger@itp.uni-tuebingen.de, manteuffel@uni-mainz.de, \\ stephan.christoph.thier@desy.de
}

ABSTRACT: We present a calculation of the next-to-leading order QCD corrections to slepton pair production in association with a jet at the LHC together with their implementation in the POWHEG BOX. For the simulation of parton-shower effects and the decays of the sleptons we employ the multi-purpose Monte-Carlo program PYTHIA. We discuss the impact of next-to-leading order QCD corrections on experimentally accessible distributions and illustrate how the parton shower can modify observables that are sensitive to QCD radiation effects. Having full control on the hard jet in the process, we provide precise predictions also for monojet analyses.

Keywords: Monte Carlo Simulations, NLO Computations

ARXIV EPRINT: 1410.3802 


\section{Contents}

1 Introduction 1

2 Technical details of the calculation 2

3 Phenomenological results and discussion 5

4 Summary and conclusions $\quad 12$

\section{Introduction}

With the start-up of the CERN Large Hadron Collider (LHC) particle physics has entered a new era, culminating in the discovery of the Higgs boson $[1,2]$. Nonetheless, we are left with a plethora of open questions pointing to the necessity of extending the Standard Model (SM) of elementary particles. A theoretically particularly appealing approach is the construction of supersymmetric (SUSY) theories that predict the existence of new particles which, before SUSY breaking, merely differ in their spin quantum numbers from the SM partners they are associated with.

As of yet, the ATLAS and CMS collaborations are providing severe exclusion limits on strongly interacting supersymmetric particles (see, e.g., refs. [3, 4]). Because of smaller production cross sections, weakly interacting supersymmetric particles are more difficult to access, but have recently gained increasing attention by both experimental collaborations [5-7]. In the context of the minimal supersymmetric extension of the Standard Model (MSSM) much effort has also been made by theorists to provide precise predictions for the production of these color neutral particles. Perturbative calculations for the pair production of the scalar partners of the leptons, the sleptons, for instance, have been ever refined during the last decades. Next-to-leading order (NLO) QCD and SUSY-QCD (SQCD) corrections to slepton pair production processes at hadron colliders ${ }^{1}$ have first been computed in [12] and [13], respectively. The latter calculation is publicly available in the format of the computer package PROSPINO [14]. Resummation effects have been considered in refs. [1518]. In refs. [19] and [20], NLO-(S)QCD corrections to slepton pair production processes have been matched with HERWIG++ [21, 22] and PYTHIA [23], respectively, making use of the POWHEG method [24, 25], an approach that allows to combine fixed-order perturbative calculations with parton-shower programs in a well-defined manner.

While parton-shower programs are capable of simulating the emission of soft and/or collinear partons in a hard-scattering event, they are not designed to account for extra hard emissions. If processes with extra hard jets in the final state, as observed frequently at the

\footnotetext{
${ }^{1}$ Precise determinations of the slepton properties could be performed at a future $e^{+} e^{-}$linear collider [810], and, with restrictions, at a muon collider [11].
} 
(a1)

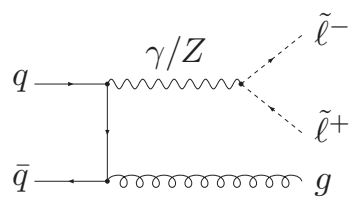

(c1)

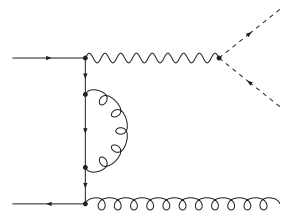

(b1)

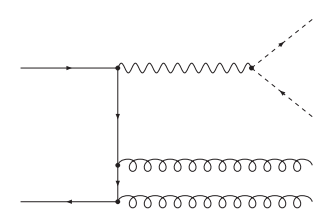

(c2)

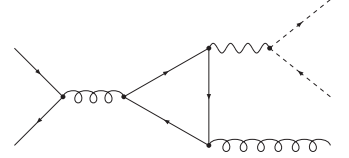

(b2)

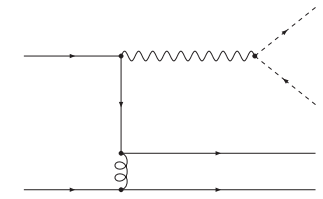

(c3)

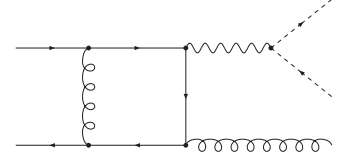

Figure 1. Representative Feynman diagrams for slepton pair production in association with a jet at leading order (a1), with an additional real parton (b1)-(b2), and with virtual SM QCD corrections (c1)-(c3).

LHC, are to be described realistically, the hard-scattering amplitudes themselves have to account for these jets. This is particularly important for the study of monojet signatures in scenarios where the decay products of the sleptons are difficult to detect. In this work we therefore provide an explicit NLO-SQCD calculation for slepton pair production in association with a hard jet. Moreover, we describe the implementation of our calculation in the POWHEG BOX [26], a repository that contains all process-independent ingredients of the POWHEG method. We discuss the implications of the NLO corrections as well as of the parton shower on experimentally accessible distributions. Comparison to previous work performed in the same framework allows us to demonstrate how the description of the hard jet gains from explicit matrix elements for the full hard-scattering process in the relevant kinematic domains.

\section{Technical details of the calculation}

Our calculation of the NLO-(S)QCD corrections to slepton pair production in association with a jet proceeds along similar lines as our previous calculation [20] for slepton pair production at the LHC in the framework of the MSSM.

At leading order, we encounter the annihilation of a massless quark-antiquark pair into an intermediate $Z$ boson or photon that in turn decays into a slepton pair, accompanied by a gluon emitted from either of the incoming partons, as shown in figure 1 (a). In addition to the process $q \bar{q} \rightarrow \tilde{\ell}^{+} \tilde{\ell}^{-} g$, crossing-related processes with a quark or antiquark and a gluon in the initial state, such as $q g \rightarrow \tilde{\ell}^{+} \tilde{\ell}^{-} q$ or $\bar{q} g \rightarrow \tilde{\ell}^{+} \tilde{\ell}-\bar{q}$, occur.

The real-emission contributions comprise scattering processes at order $\alpha_{s}^{2} \alpha^{2}$ with a slepton pair and two partons in the final state, i. e. subprocesses of the type $q \bar{q} \rightarrow \tilde{\ell}^{+} \tilde{\ell}^{-} q^{\prime} \bar{q}^{\prime}$, $q \bar{q} \rightarrow \tilde{\ell}^{+} \tilde{\ell}-q \bar{q}, q \bar{q} \rightarrow \tilde{\ell}^{+} \tilde{\ell}^{-} g g$, and all crossing-related reactions, see figure 1 (b) for two examples. In order to obtain the respective scattering amplitudes for the Born and the 
(a1)

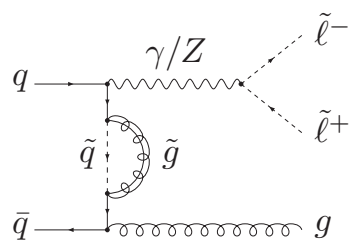

(a4)

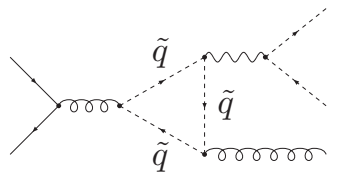

(a2)

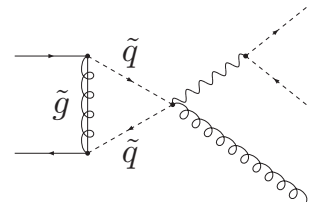

(b1)

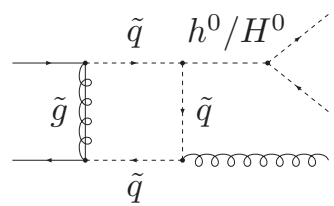

(a3)

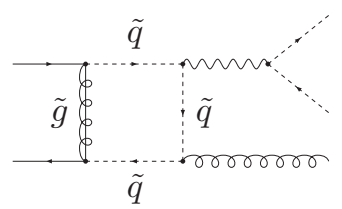

(c1)

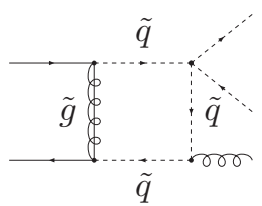

Figure 2. Representative Feynman diagrams for virtual corrections with an intermediate $Z$ boson or photon and supersymmetric particles in the loop (a1)-(a4), with an intermediate Higgs boson (b1), or with a squark-squark-slepton-slepton vertex (c1).

real-emission contributions in a format that can easily be processed by the POWHEG BOX, we made use of the build tool based on MadGraph 4 [27-29] that was first applied in ref. [30] and is now provided with the public version of the program repository. The default version of the POWHEG BOX is constrained to the Standard Model, but the code can be extended in a straightforward manner to processes involving weakly interacting ${ }^{2}$ supersymmetric $^{2}$ particles in the context of the MSSM with the help of SMadGraph [32]. The suitably adapted build tool then also provides us with the color- and spin-correlated Born amplitudes that are needed for the construction of the counterterms for IR singular configurations in the framework of the Frixione-Kunszt-Signer subtraction formalism [33].

The calculation of the virtual (S)QCD corrections is performed with the methods of ref. [20], with appropriate refinements and extensions to account for the more involved structure of slepton pair production with an extra parton in the final state. Throughout, we are using conventional dimensional regularization to handle ultraviolet (UV) and infrared (IR) divergences in a well-defined manner. For the partonic subprocesses under scrutiny, no supersymmetry restoring counterterms are required [34]. UV divergences are absorbed by a proper renormalization procedure for quark- and gluon fields as well as the strong coupling constant $\alpha_{s}$ with five active massless flavors. We work in the on-shell scheme for the renormalization of the quark- and gluon wavefunctions. For the renormalization of $\alpha_{s}$ we employ the $\overline{\mathrm{MS}}$ scheme, modified to decouple the top quark $[35,36]$ and heavy SUSY particles [37]. As a consequence, heavy-particle contributions to the counter terms for our calculation enter only through the quark wave function renormalization.

The virtual corrections can be split into four different groups. The first two groups resemble the loop corrections to the Drell-Yan plus jet process with an intermediate $Z$ boson or photon, either with SM particles, see figure 1 (c), or with supersymmetric particles in

\footnotetext{
${ }^{2}$ Let us note here that a similar extension to processes involving squarks or gluinos would require special care [31].
} 
the loops, see figure 2 (a), respectively. In the third group the sleptons originate from one of the MSSM Higgs bosons, see figure 2 (b). In the fourth group the sleptons originate from a four-particle vertex of two squarks and two sleptons, see figure 2 (c). Since we employ the Feynman-'t Hooft gauge for all gauge fields, the $Z$ boson exchange diagrams are understood to be supplemented by corresponding Goldstone contributions. In the simplest case of same-mass sleptons and absence of mixing in the squark sector, various subsets of these diagrams such as Goldstone contributions, closed squark loop contributions and contributions with a squark-squark-gluon- $Z$ vertex vanish, either diagram by diagram or in the sum.

Individual diagrams with a $Z$ boson coupling to a closed fermion loop, see figure 1 (c2), exhibit the Adler-Bell-Jackiw axial anomaly [38, 39]. To calculate its remnant in the full amplitude due to the finite top quark mass we employ two different schemes for $\gamma_{5}$ in $D \neq 4$ dimensions, that were suggested in refs. [40, 41] and [42], respectively, and find complete agreement.

For diagrams with Drell-Yan plus jet like structure, we apply the decomposition of the process $q+\bar{q} \rightarrow \gamma^{*} / Z^{*}+g$ into Lorentz structures, which we employed for the real-emission contributions to slepton pair production in ref. [20] already. Projectors constructed from this decomposition are applied to process Feynman diagrams generated with QGRAF [43] using Feynman rules from ref. [44] and in-house developed FORM [45, 46] scripts. The loop integrals obtained in this calculation are reduced with Reduze 2 [47-50], yielding expressions that contain only scalar master integrals which can be evaluated numerically with the QCDloop library [51, 52]. A second calculation of the Drell-Yan plus jet like virtual corrections based on FeynArts [53], FormCalc [54, 55], and LoopTools [52, 54] provides an independent check for these contributions. For our FormCalc calculation, we carefully implement alternative routines for the evaluation of fermion traces according to our treatment of $\gamma_{5}$. We find complete agreement between the two calculations. Virtual corrections featuring Goldstone bosons, Higgs bosons, or squark-squark-slepton-slepton vertices, are implemented in our code based on matrix elements obtained with FeynArts, FormCalc, and appropriate integral reduction formulae [56].

For the parameterization of the phase space, we adapt the implementation of ref. [57] that was originally developed for the related case of $t \bar{t} j$ production at the LHC in the POWHEG BOX. The inclusive Born cross section for slepton pair production in association with a jet is singular when the final-state parton becomes soft or collinear to an incoming parton. Once realistic acceptance cuts are imposed on the jet, such contributions are irrelevant for phenomenological applications. However, they spoil the efficiency of the program, if not handled with care. The POWHEG BOX offers two approaches for dealing with singular Born configurations: generation cuts that avoid the population of unwanted regions in phase space from the beginning, and a so-called Born-suppression factor that dampens contributions from singular regions of phase space. In order to ensure our phenomenological results do not depend on the selected procedure, we ran the code with both options. After applying a realistic transverse-momentum cut of $p_{\mathrm{T}}^{\text {jet1 }}>20 \mathrm{GeV}$ on the hardest jet at analysis level we found identical results for a setup with a generation cut of $p_{\mathrm{T}, i}^{\mathrm{gen}}>10 \mathrm{GeV}$ on the final-state parton $i$ of the underlying Born configuration and a setup with a Born 
suppression factor of the form

$$
F\left(\Phi_{n}\right)=\frac{p_{\mathrm{T}, i}^{2}}{p_{\mathrm{T}, i}^{2}+\Lambda^{2}}
$$

with $\Lambda=10 \mathrm{GeV}$. For the results presented in section 3, we employ the Born suppression factor of eq. (2.1) with $\Lambda=10 \mathrm{GeV}$ together with a mild generation cut of $p_{\mathrm{T}, i}^{\text {gen }}>1 \mathrm{GeV}$.

While our calculation represents the first complete NLO-SQCD calculation for slepton pair production in association with a jet, the related case of lepton-pair production with an associated jet in NLO QCD has been considered long ago and is available in the POWHEG BOX [58]. We compare the virtual corrections in this implementation of $p p \rightarrow \ell^{+} \ell^{-}+$jet $+X$ to the corresponding terms in an adapted version of our code. Replacing the sleptons of our calculation with leptons, adjusting couplings and input parameters, and selecting appropriate diagrams, we are able to reproduce the results of this code at representative phase-space points. Since our setup encapsulates the (renormalized) loop contributions in scalar coefficient functions which are independent of the decay of the photon or $Z$ boson, this comparison provides a very direct check on our calculation of the virtual corrections and their implementation in the POWHEG BOX.

Additional checks are based on the observation that the Born amplitudes for $p p \rightarrow$ $\tilde{\ell}^{+} \tilde{\ell}^{-}+$jet $+X$ equal the real emission amplitudes of the slepton pair production process $p p \rightarrow \tilde{\ell}^{+} \tilde{\ell}^{-}+X$. A comparison of our leading-order matrix elements for $p p \rightarrow \tilde{\ell}^{+} \tilde{\ell}^{-}+$ jet $+X$ with the real-emission amplitudes of [20] shows excellent agreement. Moreover, for differential distributions of the hardest jet we find full agreement between the two codes ran at leading order and NLO accuracy, respectively, after realistic selection cuts. This provides a powerful test on the phase space integration and the overall normalization of the new code.

\section{Phenomenological results and discussion}

Our implementation of slepton pair production in association with a jet in the POWHEG BOX will be made publicly available at http://powhegbox.mib.infn.it/. Together with the code we provide a documentation with instructions and recommended technical parameters for running the program. The interested reader is free to use the default version of the code including routines for a phenomenological analysis, or to adapt input parameters, histograms, and selection cuts to his own needs. In order to demonstrate the capability of the code, here we present results for some phenomenologically interesting setups.

We consider proton-proton collisions at the LHC with a center-of-mass energy of $\sqrt{s}=$ $14 \mathrm{TeV}$. For the parton-distribution functions of the proton we use the NLO-QCD set of the MSTW2008 parameterization [59], as implemented in the LHAPDF library [60]. Factorization and renormalization scales are set to $\mu_{R}=\mu_{F}=\mu_{0}$ with $\mu_{0}=2 m_{\tilde{\ell}}$, unless explicitly stated otherwise. Statistical uncertainties are negligible for all results presented here.

All required SM and MSSM parameters are provided in a file complying with the SUSY Les Houches Accord (SLHA) [61, 62]. They are processed by routines based on MadGraph 4, which calculate the dependent parameters and all particle couplings. As electroweak input parameters we are using the mass of the $Z$ boson, $m_{Z}=91.1876 \mathrm{GeV}$, the electromagnetic 
coupling, $\alpha\left(m_{Z}\right)=1 / 127.944$, and the Fermi constant, $G_{\mathrm{F}}=1.1663787 \times 10^{-5} \mathrm{GeV}^{-2}$. The top quark mass is set to $m_{t}=173.07 \mathrm{GeV}$, all other quark masses are neglected.

Our default setup for $p p \rightarrow \tilde{\ell}^{+} \tilde{\ell}^{-}+$jet $+X$ features selectrons or smuons with a mass

$$
m_{\tilde{\ell}}=350 \mathrm{GeV}
$$

that lies above current exclusion limits by ATLAS [63] and CMS [64] irrespective of the masses of potential decay products. We do not consider sleptons of the third generation but, for our representative analysis, restrict ourselves to electrically charged left-handed sleptons of one of the first two generations (no sum) without slepton mixing. The mass of the lightest neutralino $\tilde{\chi}_{0}^{1}$ is taken as

$$
m_{\tilde{\chi}_{1}^{0}}=100 \mathrm{GeV}
$$

while we assume the other neutralinos and the charginos to be heavier than the sleptons. In this scenario, sleptons exclusively decay into a lepton and the lightest neutralino. We simulate these decays as well as parton-shower effects with the help of PYTHIA 6.4.25. Throughout, we switch off QED radiation, underlying event and hadronization effects.

Partons in the final state are recombined into jets with the help of the anti- $k_{T}$ algorithm of ref. [65], as implemented in the FASTJET package [66, 67], with a resolution parameter of $R=0.4$. For our numerical studies we require the presence of at least one jet with

$$
p_{\mathrm{T}}^{\text {jet1 }}>20 \mathrm{GeV}, \quad\left|y^{\text {jet1 }}\right|<4.5 .
$$

In the setting described above we compute the LO cross section for $p p \rightarrow \tilde{\ell}^{+} \tilde{\ell}^{-}+$jet $+X$ and find

$$
\sigma_{\mathrm{LO}}(\tilde{\ell} \tilde{\jmath} j)=1.624 \mathrm{fb} .
$$

Based on the experience from slepton pair production [20] and Drell-Yan plus jet production [68], where virtual corrections with supersymmetric particles in the loops were found to be very small compared to the other contributions, we test the impact of different groups of virtual corrections separately. Considering just Drell-Yan plus jet like SM contributions we find for the NLO cross section

$$
\sigma_{\mathrm{NLO}, \mathrm{DY}(\mathrm{SM})}(\tilde{\ell} \tilde{\ell} j)=1.826 \mathrm{fb} .
$$

Including also supersymmetric Drell-Yan plus jet like corrections, we find that even for a light common squark mass of $m_{\tilde{q}}=500 \mathrm{GeV}$ and a light gluino mass of $m_{\tilde{g}}=700 \mathrm{GeV}$ the cross section changes only slightly, $\sigma_{\mathrm{NLO}, \mathrm{DY}(\mathrm{SM}+\mathrm{SUSY})}(\tilde{\ell} \tilde{\ell} j)=1.847 \mathrm{fb}$. The remaining corrections with either a Higgs boson of mass $m_{h^{0}}=126 \mathrm{GeV}$ or $m_{H^{0}}=400 \mathrm{GeV}$ or with a squark-squark-slepton-slepton vertex are found to be completely negligible at this level of accuracy. Consequently, we neglect such contributions in our phenomenological study, only the virtual corrections generated by diagrams with QCD loops, see figure 1 (c), are included. In this way, we reduce both the number of free parameters in our setup and the execution time of our code. 

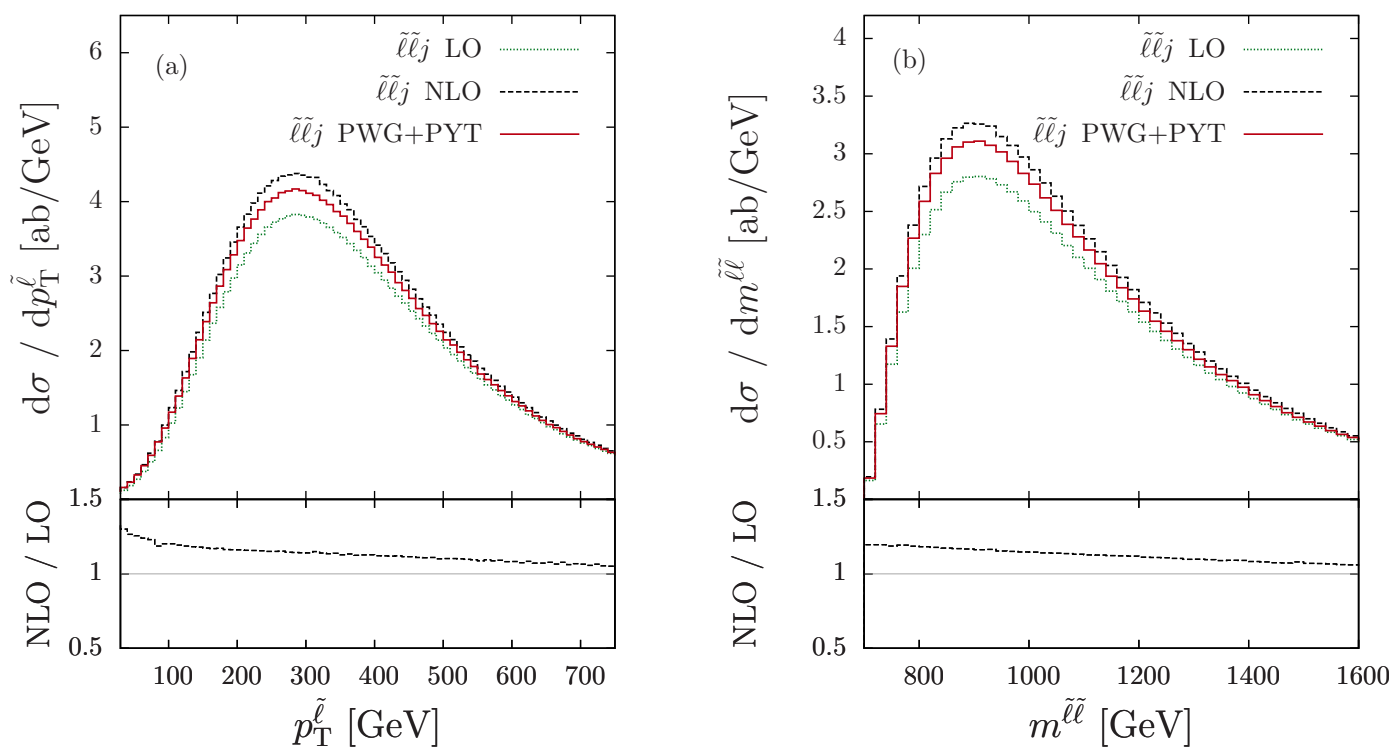

Figure 3. Transverse momentum of the negatively charged slepton (a) and invariant-mass distribution of the slepton pair (b) at LO (green dotted), NLO-QCD (black dashed), and with POWHEG+PYTHIA (red solid) in our default setup for $p p \rightarrow \tilde{\ell}^{+} \tilde{\ell}^{-}+$jet $+X$.

Distributions related to the sleptons receive sizable corrections compared to LO results when NLO contributions are included, and the parton shower gives rise to further noticable effects. In figure 3 this is illustrated for the transverse momentum of the negatively charged slepton and the invariant mass distribution of the slepton pair in $p p \rightarrow \tilde{\ell}^{+} \tilde{\ell}^{-}+$jet $+X$.

In ref. [20] we have investigated inclusive slepton pair production, $p p \rightarrow \tilde{\ell}^{+} \tilde{\ell}^{-}+X$, at NLO-QCD, matched with PYTHIA via the POWHEG approach. In that work, distributions related to the hardest jet could be accounted for only at leading-order, whereas our new implementation of $p p \rightarrow \tilde{\ell}^{+} \tilde{\ell}^{-}+$jet $+X$ provides full NLO-QCD accuracy for this class of observables. The transverse momentum distributions of the hardest jet in the two approaches are shown in figure 4 (a). Scale uncertainties in this jet distribution, obtained by varying the factorization and renormalization scales independently in the range $0.5 \mu_{0} \leq$ $\mu_{\mathrm{R}}, \mu_{\mathrm{F}} \leq 2 \mu_{0}$, are provided in figure 5 . The widths of the bands can be considered as rough indicators for the theoretical uncertainties of the respective predictions. Clearly, providing matrix elements at NLO-QCD accuracy for $\tilde{\ell}^{+} \tilde{\ell}^{-}+$jet final states much improves the uncertainty associated with jet distributions emphasizing the need for such a calculation, in particular for observables related to the jet.

While in $p p \rightarrow \tilde{\ell}^{+} \tilde{\ell}^{-}+X$ any jet apart from the hardest can be simulated by the parton shower only, in $p p \rightarrow \tilde{\ell}^{+} \tilde{\ell}^{-}+$jet $+X$ a second hard jet can be accounted for by the real-emission contributions of the hard matrix elements. Figure 4 (b) shows the transverse momentum distribution of the second-hardest jet as obtained with the respective programs for $p p \rightarrow \tilde{\ell}^{+} \tilde{\ell}^{-}+X$ and $p p \rightarrow \tilde{\ell}^{+} \tilde{\ell}^{-}+$jet $+X$. Clearly, the inclusion of contributions from the matrix element in $p p \rightarrow \tilde{\ell}^{+} \tilde{\ell}^{-}+$jet $+X$ gives a better description of hard jet configurations, while the Sudakov factor provided by the POWHEG+PYTHIA implementation provides the expected suppression of contributions with low transverse momenta. 

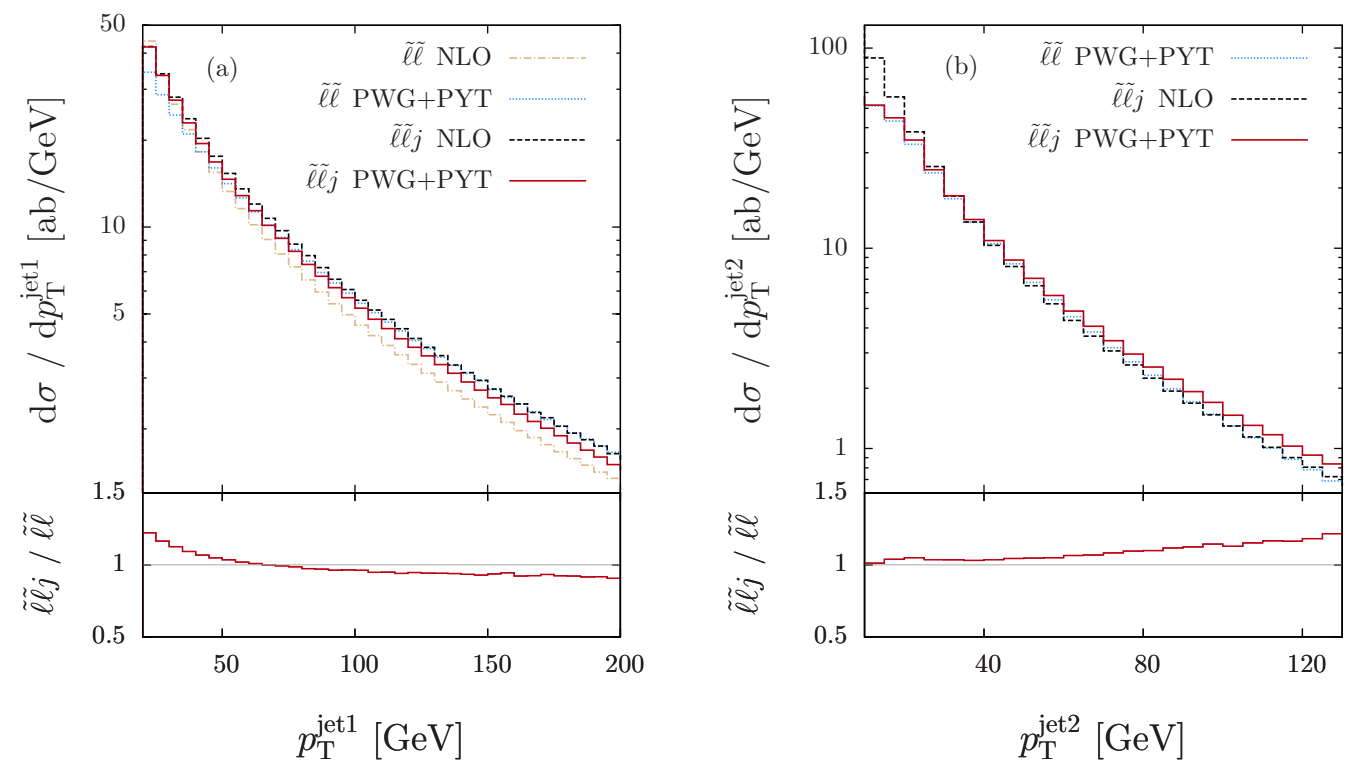

Figure 4. Transverse momentum of the hardest jet (a) and the second-hardest jet (b) for our default setup in $p p \rightarrow \tilde{\ell}^{+} \tilde{\ell}^{-}+X$ at NLO (beige dot-dashed), $p p \rightarrow \tilde{\ell}^{+} \tilde{\ell}^{-}+X$ with POWHEG+PYTHIA (blue dotted), $p p \rightarrow \tilde{\ell}^{+} \tilde{\ell}^{-}+$jet $+X$ at NLO (black dashed), and $p p \rightarrow \tilde{\ell}^{+} \tilde{\ell}^{-}+$jet $+X$ with POWHEG+PYTHIA (red solid). The respective ratios of the POWHEG+PYTHIA results for $p p \rightarrow \tilde{\ell}^{+} \tilde{\ell}^{-}+$jet $+X$ and $p p \rightarrow \tilde{\ell}^{+} \tilde{\ell}^{-}+X$ are shown in the lower panels.

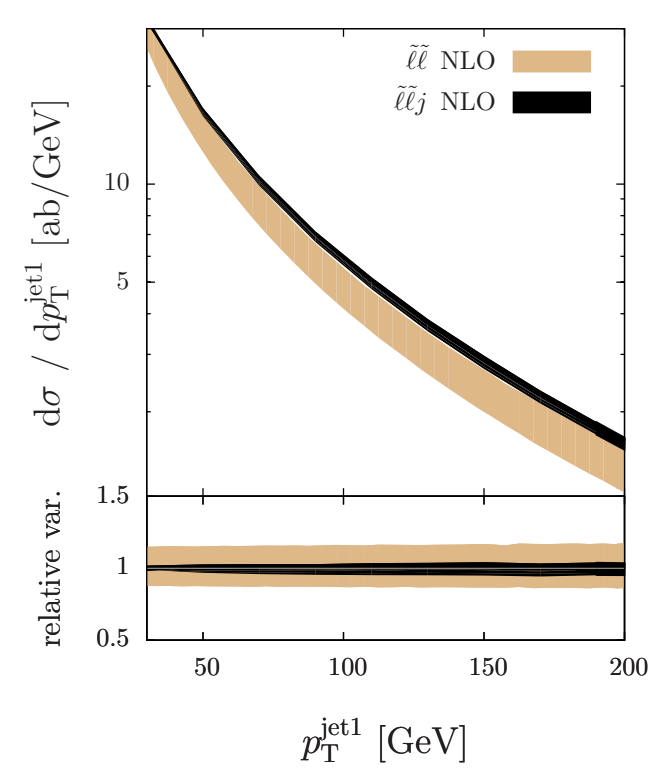

Figure 5. Scale variation for the transverse momentum distribution of the hardest jet at NLO for $p p \rightarrow \tilde{\ell}^{+} \tilde{\ell}^{-}+X$ (beige) and $p p \rightarrow \tilde{\ell}^{+} \tilde{\ell}^{-}+$jet $+X$ (black) with $0.5 \mu_{0} \leq \mu_{\mathrm{R}}, \mu_{F} \leq 2 \mu_{0}$ (upper panel). The respective variations relative to the central scale choice $\mu_{0}$ are given in the lower panel. 

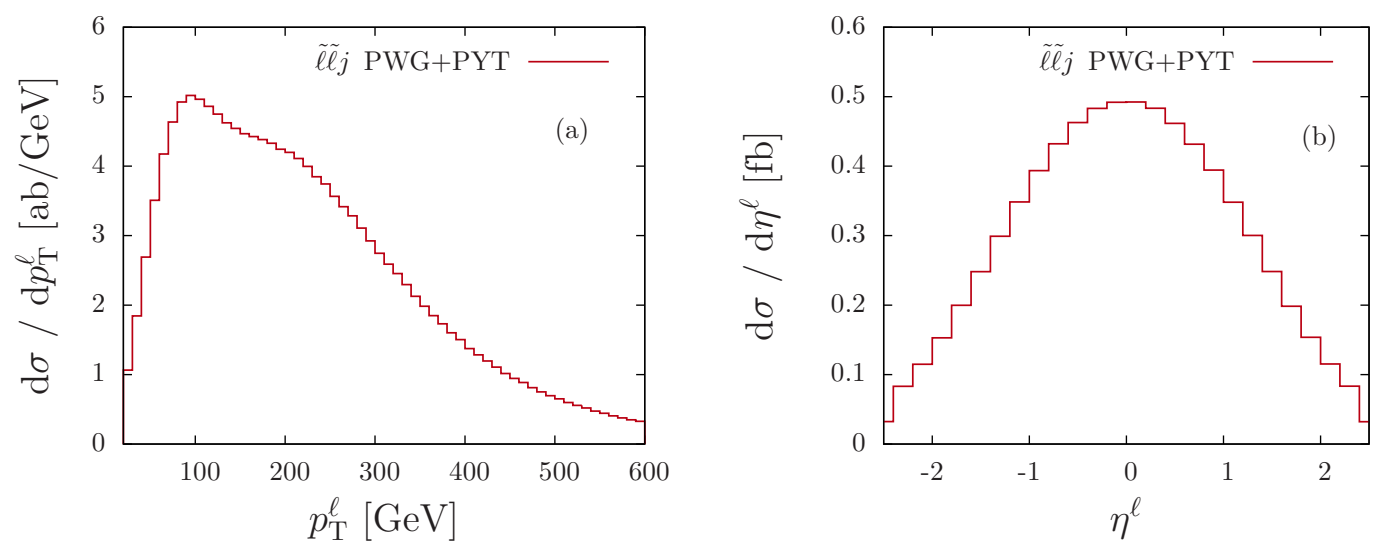

Figure 6. Transverse momentum (a) and rapidity distribution (b) of the hardest negatively charged lepton in $p p \rightarrow \tilde{\ell}^{+} \tilde{\ell}^{-}+$jet $+X$ (red solid) with POWHEG+PYTHIA.

A realistic analysis of slepton pair production processes requires access to the kinematic properties of the decay products of the heavy SUSY particles. Such decays can be conveniently simulated by PYTHIA. To illustrate the capability of our code that handles decays of the sleptons via an interface to PYTHIA, we consider a setup with two oppositely charged hard, central leptons,

$$
p_{\mathrm{T}}^{\ell}>20 \mathrm{GeV}, \quad\left|\eta^{\ell}\right|<2.5
$$

in the presence of a hard jet, fulfilling eq. (3.3). The leptons are required to be wellseparated from each other and from the jets, in the rapidity-azimuthal angle plane,

$$
\Delta R_{\ell \ell}>0.4, \quad \Delta R_{\ell j}>0.4
$$

In figure 6 we show the transverse momentum and rapidity distributions of the hardest negatively charged lepton in $p p \rightarrow \tilde{\ell}^{+} \tilde{\ell}^{-}+$jet $+X$ as obtained with POWHEG+PYTHIA.

Since we are having full access to the kinematics of the decay leptons, we can also provide distributions for sophisticated observables that are typically used in SUSY analyses for an optimal signal selection in the presence of background processes with a priori large event rates. In ref. [69], super-razor variables have been introduced as a means to improve searches for weakly interacting new particles that are produced in pairs at the LHC, such as charginos and sleptons. Super-razor variables are constructed by approximate boosts to the center-of-mass frame of the slepton-pair system, followed by boosts to the slepton decay frames, see ref. [69] for details. In the presence of QCD radiation, boosts against the jet directions are included. Due to this construction, extra jets in an event do not alter the shape of super-razor variables. The $\tilde{\ell}^{+} \tilde{\ell}^{-}+X$ and $\tilde{\ell}^{+} \tilde{\ell}^{-}+$jet $+X$ processes thus exhibit super-razor variables of the same shape.

The super-razor variable $M_{\Delta}^{R}$ contains information about the mass differences involved in the pair production and subsequent decay. For slepton pair production processes, the 

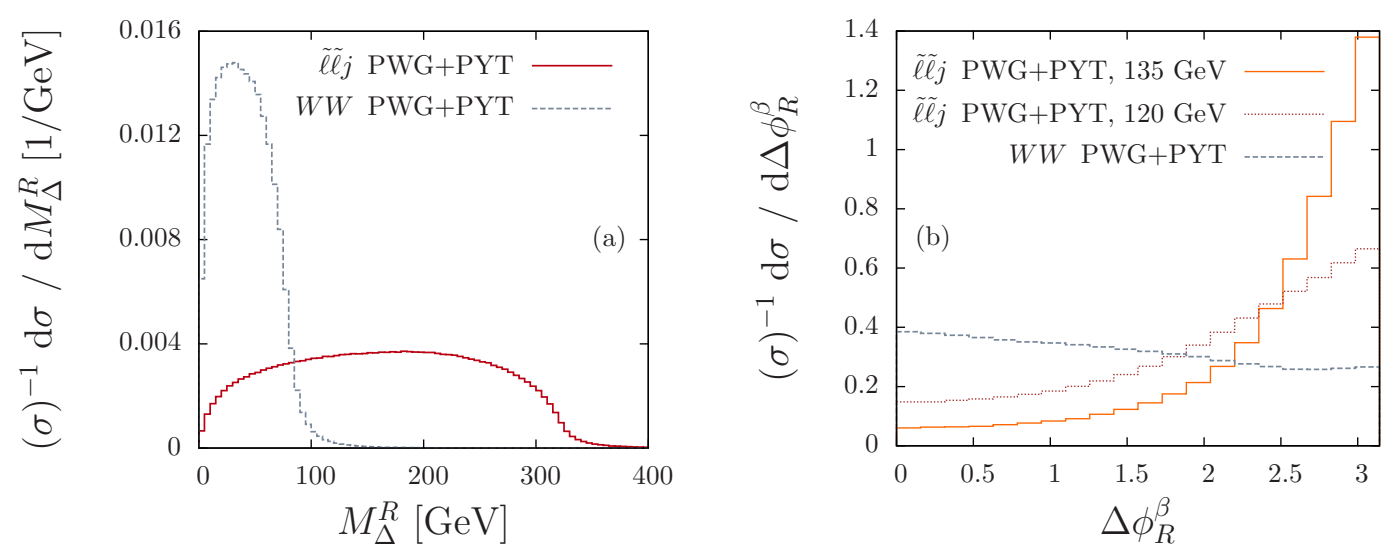

Figure 7. Left panel: normalized distribution of the super-razor variable $M_{\Delta}^{R}$ in our default MSSM setup, $m_{\tilde{\ell}}=350 \mathrm{GeV}$, for $p p \rightarrow \tilde{\ell}^{+} \tilde{\ell}^{-}+$jet $+X$ (red solid) and for $p p \rightarrow W^{+} W^{-}+X$ (grey dashed). Right panel: normalized distribution of the super-razor variable $\Delta \phi_{R}^{\beta}$ for $p p \rightarrow \tilde{\ell}^{+} \tilde{\ell}^{-}+$jet $+X$ in a light slepton setup, $m_{\tilde{\ell}}=150 \mathrm{GeV}$, with $m_{\tilde{\chi}_{1}^{0}}=135 \mathrm{GeV}$ (orange solid), $m_{\tilde{\chi}_{1}^{0}}=120 \mathrm{GeV}$ (brown dotted) and for $p p \rightarrow W^{+} W^{-}+X$ (grey dashed).

$M_{\Delta}^{R}$ distribution drops rapidly at $M_{\Delta}^{R}=M_{\Delta}$, with

$$
M_{\Delta}=\frac{m_{\tilde{\ell}}^{2}-m_{\tilde{\chi}}^{2}}{m_{\tilde{\ell}}} .
$$

Background processes do not contain information about that mass scale, and thus do not exhibit a pronounced edge at the value of $M_{\Delta}^{R}$ characterized by the mass difference between slepton and neutralino. Applying the cuts of eqs. (3.3), (3.6), (3.7), in figure 7 (a) we show the $M_{\Delta}^{R}$ distribution of the $p p \rightarrow \tilde{\ell}^{+} \tilde{\ell}^{-}+$jet $+X$ signal process in comparison to the $p p \rightarrow W^{+} W^{-}+X$ background, restricting ourselves to decays of the $W$-bosons into firstgeneration lepton-neutrino pairs. The results for the background process we have obtained with the help of the corresponding POWHEG BOX implementation [70].

Additional information on the kinematics of the reaction is provided by angular variables, such as $\Delta \phi_{R}^{\beta}$, that in the super-razor approach is constructed from the boost direction and the momenta of the visible decay particles. Such angular variables are particularly powerful in scenarios where the mass difference of the sleptons and the neutralinos they decay into is small. For a mass difference of $50 \mathrm{GeV}$ or less, sleptons as light as $100 \mathrm{GeV}$ are not yet excluded. We study this case in a light slepton setup with a slepton mass of $m_{\tilde{\ell}}=150 \mathrm{GeV}$ and different values of the lightest neutralino mass $m_{\tilde{\chi}_{1}^{0}}$ close to $m_{\tilde{\ell}}$ and, again, the cuts of eqs. (3.3), (3.6), (3.7). The discriminatory power of $\Delta \phi_{R}^{\beta}$ in the light slepton setup is illustrated in figure 7 (b) for neutralino masses of $m_{\tilde{\chi}_{1}^{0}}=120 \mathrm{GeV}$ and $m_{\tilde{\chi}_{1}^{0}}=135 \mathrm{GeV}$, respectively. While for the signal process the leptons tend to be aligned with each other, opposite to the boost direction, resulting in a peak of the $\Delta \phi_{R}^{\beta}$ distribution around $\pi$, especially for a small mass difference between slepton and neutralino, the $W^{+} W^{-}$ background does not exhibit such a correlation, but features a rather flat distribution. 

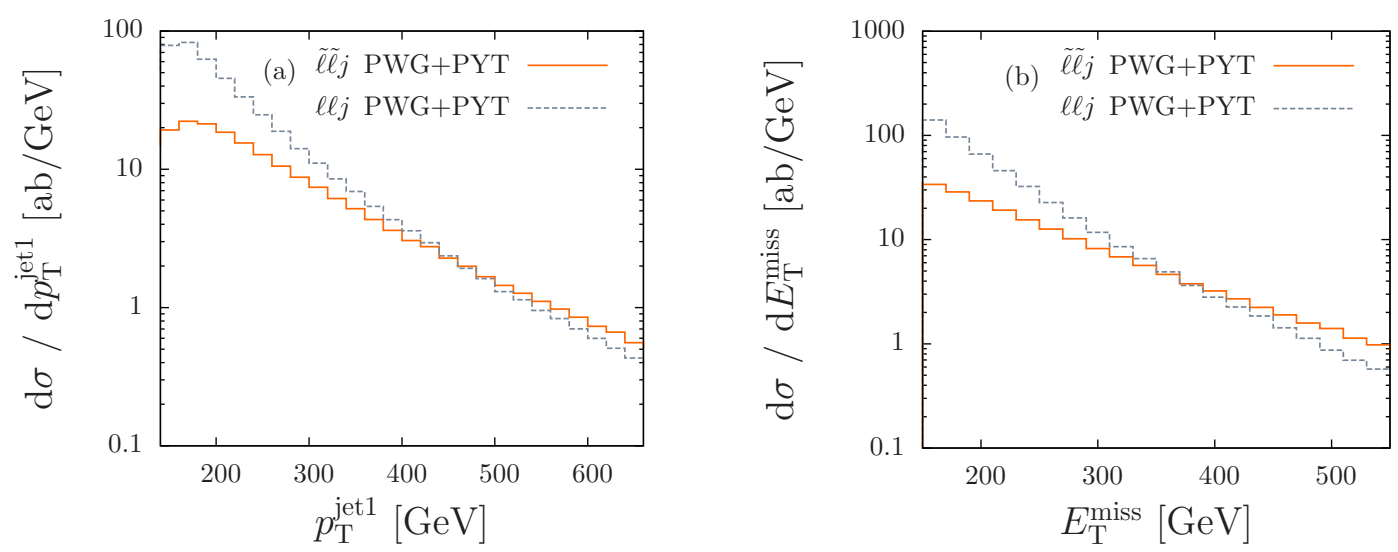

Figure 8. Transverse momentum of the hardest jet (a) and missing transverse energy (b) for $p p \rightarrow$ $\tilde{\ell}^{+} \tilde{\ell}^{-}+$jet $+X$ (orange solid) and $p p \rightarrow \ell^{+} \ell^{-}+$jet $+X$ (grey dashed) in our monojet analysis setup.

In SUSY scenarios where the masses of the sleptons and neutralinos are almost degenerate, slepton decays produce neutralinos and very soft leptons that easily escape detection. The tell-tale signature of such reactions is the missing transverse energy associated with the neutralinos accompanied by hard QCD radiation resulting in a monojet configuration. To make use of this signature, an accurate, matrix-element based description of the hard jet is mandatory. We illustrate the capability of our code for $p p \rightarrow \tilde{\ell}^{+} \tilde{\ell}^{-}+$jet $+X$ to serve that purpose in the light slepton scenario for neutralinos with a mass of $m_{\tilde{\chi}_{1}^{0}}=135 \mathrm{GeV}$, that are almost degenerate with the $m_{\tilde{\ell}}=150 \mathrm{GeV}$ sleptons.

Following the strategies for monojet searches as a tool for the discovery of only weakly interacting SUSY particles at hadron colliders presented in [71-74], we require at least one hard jet,

$$
p_{\mathrm{T}}^{\text {jet1 }}>120 \mathrm{GeV}, \quad\left|y^{\text {jet1 }}\right|<4.5,
$$

and large missing energy, computed from all observed tracks in an event,

$$
E_{T}^{\text {miss }}>150 \mathrm{GeV} \text {. }
$$

The cuts of eqs. (3.9)-(3.10) are constructed to account for efficiency requirements in the missing energy triggers of the LHC experiments and to suppress background contributions that are a priori dominant at low transverse momenta, such as weak boson production in association with a jet. To sketch the general features of such background processes, we use the POWHEG BOX implementation [58] for $p p \rightarrow Z+$ jet $+X$ in the $Z \rightarrow e^{+} e^{-}$decay mode, assuming that the decay leptons escape detection and give thus rise to missing transverse energy. Figure 8 shows the transverse momentum and the missing transverse energy distributions of the signal and background processes within the cuts of eqs. (3.9)(3.10). In each case, the signal contribution takes over in the tail of the distribution, thus confirming the desired impact of hard transverse momentum cuts on the signal significance. We note that a full signal-to-background analysis would require considering all possible decay modes of the $Z$ boson and, moreover, additional background processes such as $p p \rightarrow$ $W+$ jet $+X$. Such a detailed analysis is, however, far beyond the scope of this work, where 
we intend to simply illustrate the general benefit of monojet analyses in the context of slepton pair production processes and point out the usefulness of our code for such studies.

\section{Summary and conclusions}

In this work we have presented an NLO-QCD calculation for slepton pair production in association with a hard jet at the LHC, and its matching with parton-shower programs in the framework of the POWHEG BOX. While the reader is free to download the publicly available computer package and use it for applications of his own, we have presented numerical results for selected phenomenological applications to illustrate the capability of our code and demonstrate the impact of radiative corrections and parton-shower effects on realistic analyses.

We found that the NLO-QCD corrections to $p p \rightarrow \tilde{\ell}^{+} \tilde{\ell}^{-}+$jet $+X$ are sizable, thus crucially requiring hard matrix elements at order $\mathcal{O}\left(\alpha_{s}^{2} \alpha^{2}\right)$ in order to ensure sufficient accuracy for cross sections and distributions, in particular for observables that are sensitive to the emission of hard jets. In addition, parton-shower effects can significantly modify the pattern of jet emission and, via jet-specific selection cuts, also noticeably affect distributions of the sleptons and their decay products.

The matching of the NLO calculation with PYTHIA allows us not only to account for parton-shower effects, but also provides us with a convenient tool for the simulation of slepton decays. This feature puts us into a position to compute observables constructed from the momenta of decay products, such as the so-called super-razor variables. Having full control on the hard jet in $p p \rightarrow \tilde{\ell}^{+} \tilde{\ell}^{-}+$jet $+X$, we can also provide reliable predictions for monojet analyses which have been developed for the extraction of SUSY signatures that are difficult to detect by other means.

\section{Acknowledgments}

We are grateful to José Zurita for many valuable discussions. We thank Stefan Berge and Stefan Weinzierl for clarifying discussions, and Thomas Hahn and Emanuele Re for useful comments. This work was supported in part by the Institutional Strategy of the University of Tübingen (DFG, ZUK 63), by the GRK Symmetry Breaking (DFG/GRK 1581) of the German Research Foundation (DFG), the DFG Grant JA 1954/1, and by the PRISMA Cluster of Excellence.

Open Access. This article is distributed under the terms of the Creative Commons Attribution License (CC-BY 4.0), which permits any use, distribution and reproduction in any medium, provided the original author(s) and source are credited.

\section{References}

[1] ATLAS collaboration, Observation of a new particle in the search for the standard model Higgs boson with the ATLAS detector at the LHC, Phys. Lett. B 716 (2012) 1 [arXiv:1207.7214] [INSPIRE]. 
[2] CMS collaboration, Observation of a new boson at a mass of $125 \mathrm{GeV}$ with the CMS experiment at the LHC, Phys. Lett. B $\mathbf{7 1 6}$ (2012) 30 [arXiv:1207.7235] [INSPIRE].

[3] ATLAS physics results webpage, https://twiki.cern.ch/twiki/bin/view/AtlasPublic.

[4] CMS physics results webpage, https://twiki.cern.ch/twiki/bin/view/CMSPublic/PhysicsResults.

[5] ATLAS collaboration, Search for direct slepton and gaugino production in final states with two leptons and missing transverse momentum with the ATLAS detector in pp collisions at $\sqrt{s}=7$ TeV, Phys. Lett. B 718 (2013) 879 [arXiv:1208.2884] [INSPIRE].

[6] ATLAS collaboration, Search for direct production of charginos and neutralinos in events with three leptons and missing transverse momentum in $\sqrt{s}=7 \mathrm{TeV}$ pp collisions with the ATLAS detector, Phys. Lett. B 718 (2013) 841 [arXiv:1208.3144] [INSPIRE].

[7] CMS collaboration, Search for electroweak production of charginos and neutralinos using leptonic final states in pp collisions at $\sqrt{s}=7$ TeV, JHEP 11 (2012) 147 [arXiv:1209.6620] [INSPIRE].

[8] H.-U. Martyn and G.A. Blair, Determination of sparticle masses and SUSY parameters, hep-ph/9910416 [INSPIRE].

[9] A. Freitas, A. von Manteuffel and P.M. Zerwas, Slepton production at $e^{+} e^{-}$and $e^{-} e^{-}$linear colliders, Eur. Phys. J. C 34 (2004) 487 [hep-ph/0310182] [INSPIRE].

[10] A. Freitas, A. von Manteuffel and P.M. Zerwas, Slepton production at $e^{+} e^{-}$and $e^{-} e^{-}$linear colliders: addendum, Eur. Phys. J. C 40 (2005) 435 [hep-ph/0408341] [INSPIRE].

[11] A. Freitas, Feasibility of slepton precision measurements at a muon collider, arXiv:1107.3853 [INSPIRE].

[12] H. Baer, B.W. Harris and M.H. Reno, Next-to-leading order slepton pair production at hadron colliders, Phys. Rev. D 57 (1998) 5871 [hep-ph/9712315] [INSPIRE].

[13] W. Beenakker et al., The production of charginos/neutralinos and sleptons at hadron colliders, Phys. Rev. Lett. 83 (1999) 3780 [Erratum ibid. 100 (2008) 029901] [hep-ph/9906298] [INSPIRE].

[14] W. Beenakker, R. Hopker and M. Spira, PROSPINO: a program for the production of supersymmetric particles in next-to-leading order QCD, hep-ph/9611232 [INSPIRE].

[15] G. Bozzi, B. Fuks and M. Klasen, Transverse-momentum resummation for slepton-pair production at the CERN LHC, Phys. Rev. D 74 (2006) 015001 [hep-ph/0603074] [INSPIRE].

[16] G. Bozzi, B. Fuks and M. Klasen, Threshold resummation for slepton-pair production at hadron colliders, Nucl. Phys. B 777 (2007) 157 [hep-ph/0701202] [INSPIRE].

[17] G. Bozzi, B. Fuks and M. Klasen, Joint resummation for slepton pair production at hadron colliders, Nucl. Phys. B 794 (2008) 46 [arXiv:0709.3057] [inSPIRE].

[18] A. Broggio, M. Neubert and L. Vernazza, Soft-gluon resummation for slepton-pair production at hadron colliders, JHEP 05 (2012) 151 [arXiv:1111.6624] [INSPIRE].

[19] I. Fridman-Rojas and P. Richardson, Next-to-leading order simulation of slepton pair production, arXiv: 1208.0279 [INSPIRE].

[20] B. Jager, A. von Manteuffel and S. Thier, Slepton pair production in the POWHEG BOX, JHEP 10 (2012) 130 [arXiv:1208.2953] [INSPIRE]. 
[21] G. Marchesini et al., HERWIG: a Monte Carlo event generator for simulating hadron emission reactions with interfering gluons. Version 5.1 - April 1991,

Comput. Phys. Commun. 67 (1992) 465 [INSPIRE].

[22] G. Corcella et al., HERWIG 6: an event generator for hadron emission reactions with interfering gluons (including supersymmetric processes), JHEP 01 (2001) 010 [hep-ph/0011363] [INSPIRE].

[23] T. Sjöstrand, S. Mrenna and P.Z. Skands, PYTHIA 6.4 physics and manual, JHEP 05 (2006) 026 [hep-ph/0603175] [INSPIRE].

[24] P. Nason, A new method for combining NLO QCD with shower Monte Carlo algorithms, JHEP 11 (2004) 040 [hep-ph/0409146] [INSPIRE].

[25] S. Frixione, P. Nason and C. Oleari, Matching NLO QCD computations with parton shower simulations: the POWHEG method, JHEP 11 (2007) 070 [arXiv:0709.2092] [INSPIRE].

[26] S. Alioli, P. Nason, C. Oleari and E. Re, A general framework for implementing NLO calculations in shower Monte Carlo programs: the POWHEG BOX, JHEP 06 (2010) 043 [arXiv: 1002.2581] [INSPIRE].

[27] H. Murayama, I. Watanabe and K. Hagiwara, HELAS: HELicity amplitude subroutines for Feynman diagram evaluations, KEK-91-11, Japan (1992) [INSPIRE].

[28] T. Stelzer and W.F. Long, Automatic generation of tree level helicity amplitudes, Comput. Phys. Commun. 81 (1994) 357 [hep-ph/9401258] [INSPIRE].

[29] J. Alwall et al., MadGraph/MadEvent v4: the new web generation, JHEP 09 (2007) 028 [arXiv:0706.2334] [INSPIRE].

[30] J.M. Campbell et al., NLO Higgs boson production plus one and two jets using the POWHEG BOX, MadGraph4 and MCFM, JHEP 07 (2012) 092 [arXiv: 1202.5475] [INSPIRE].

[31] R. Gavin et al., Matching squark pair production at NLO with parton showers, JHEP 10 (2013) 187 [arXiv:1305.4061] [INSPIRE].

[32] G.-C. Cho et al., Weak boson fusion production of supersymmetric particles at the CERN LHC, Phys. Rev. D 73 (2006) 054002 [hep-ph/0601063] [INSPIRE].

[33] S. Frixione, Z. Kunszt and A. Signer, Three jet cross-sections to next-to-leading order, Nucl. Phys. B 467 (1996) 399 [hep-ph/9512328] [INSPIRE].

[34] W. Hollik and D. Stöckinger, Regularization and supersymmetry restoring counterterms in supersymmetric QCD, Eur. Phys. J. C 20 (2001) 105 [hep-ph/0103009] [InSPIRE].

[35] J.C. Collins, F. Wilczek and A. Zee, Low-energy manifestations of heavy particles: application to the neutral current, Phys. Rev. D 18 (1978) 242 [INSPIRE].

[36] P. Nason, S. Dawson and R.K. Ellis, The one particle inclusive differential cross-section for heavy quark production in hadronic collisions, Nucl. Phys. B 327 (1989) 49 [Erratum ibid. B 335 (1990) 260] [INSPIRE].

[37] S. Berge, W. Hollik, W.M. Mosle and D. Wackeroth, SUSY QCD one-loop effects in (un)polarized top-pair production at hadron colliders, Phys. Rev. D 76 (2007) 034016 [hep-ph/0703016] [INSPIRE].

[38] S.L. Adler, Axial vector vertex in spinor electrodynamics, Phys. Rev. 177 (1969) 2426 [INSPIRE]. 
[39] J.S. Bell and R. Jackiw, A PCAC puzzle: $\pi^{0} \rightarrow \gamma \gamma$ in the $\sigma$-model, Nuovo Cim. A 60 (1969) 47 [InSPIRE].

[40] D. Kreimer, The $\gamma_{5}$ problem and anomalies: a Clifford algebra approach, Phys. Lett. B 237 (1990) 59 [INSPIRE].

[41] J.G. Korner, D. Kreimer and K. Schilcher, A practicable $\gamma_{5}$ scheme in dimensional regularization, Z. Phys. C 54 (1992) 503 [INSPIRE].

[42] S.A. Larin, The renormalization of the axial anomaly in dimensional regularization, Phys. Lett. B 303 (1993) 113 [hep-ph/9302240] [INSPIRE].

[43] P. Nogueira, Automatic Feynman graph generation, J. Comput. Phys. 105 (1993) 279 [INSPIRE].

[44] J. Rosiek, Complete set of Feynman rules for the MSSM: erratum, hep-ph/9511250 [INSPIRE].

[45] J.A.M. Vermaseren, New features of FORM, math-ph/0010025 [INSPIRE].

[46] J. Kuipers, T. Ueda, J.A.M. Vermaseren and J. Vollinga, FORM version 4.0, Comput. Phys. Commun. 184 (2013) 1453 [arXiv:1203.6543] [INSPIRE].

[47] C. Studerus, Reduze-Feynman integral reduction in $\mathrm{C}++$, Comput. Phys. Commun. 181 (2010) 1293 [arXiv:0912.2546] [INSPIRE].

[48] A. von Manteuffel and C. Studerus, Reduze 2 - distributed Feynman integral reduction, arXiv: 1201.4330 [INSPIRE].

[49] C. Bauer, A. Frink and R. Kreckel, Introduction to the GiNaC framework for symbolic computation within the C++ programming language, J. Symbol. Comput. 33 (2002) 1 [cs.sc/0004015].

[50] R.H. Lewis, Computer algebra system Fermat webpage, http://www.bway.net/ lewis.

[51] R.K. Ellis and G. Zanderighi, Scalar one-loop integrals for QCD, JHEP 02 (2008) 002 [arXiv:0712.1851] [INSPIRE].

[52] G.J. van Oldenborgh, FF: a package to evaluate one loop Feynman diagrams, Comput. Phys. Commun. 66 (1991) 1 [INSPIRE].

[53] T. Hahn, Generating Feynman diagrams and amplitudes with FeynArts 3, Comput. Phys. Commun. 140 (2001) 418 [hep-ph/0012260] [INSPIRE].

[54] T. Hahn and M. Pérez-Victoria, Automatized one loop calculations in four-dimensions and D-dimensions, Comput. Phys. Commun. 118 (1999) 153 [hep-ph/9807565] [INSPIRE].

[55] T. Hahn, A Mathematica interface for FormCalc-generated code, Comput. Phys. Commun. 178 (2008) 217 [hep-ph/0611273] [INSPIRE].

[56] A. Denner and S. Dittmaier, Reduction of one loop tensor five point integrals, Nucl. Phys. B 658 (2003) 175 [hep-ph/0212259] [INSPIRE].

[57] S. Alioli, S.-O. Moch and P. Uwer, Hadronic top-quark pair-production with one jet and parton showering, JHEP 01 (2012) 137 [arXiv:1110.5251] [INSPIRE].

[58] S. Alioli, P. Nason, C. Oleari and E. Re, Vector boson plus one jet production in POWHEG, JHEP 01 (2011) 095 [arXiv: 1009.5594] [INSPIRE].

[59] A.D. Martin, W.J. Stirling, R.S. Thorne and G. Watt, Parton distributions for the LHC, Eur. Phys. J. C 63 (2009) 189 [arXiv:0901.0002] [INSPIRE]. 
[60] M.R. Whalley, D. Bourilkov and R.C. Group, The Les Houches accord PDFs (LHAPDF) and LHAGLUE, hep-ph/0508110 [INSPIRE].

[61] P.Z. Skands et al., SUSY Les Houches accord: interfacing SUSY spectrum calculators, decay packages and event generators, JHEP 07 (2004) 036 [hep-ph/0311123] [INSPIRE].

[62] B.C. Allanach et al., SUSY Les Houches accord 2, Comput. Phys. Commun. 180 (2009) 8 [arXiv:0801.0045] [INSPIRE].

[63] ATLAS collaboration, Search for direct production of charginos, neutralinos and sleptons in final states with two leptons and missing transverse momentum in pp collisions at $\sqrt{s}=8 \mathrm{TeV}$ with the ATLAS detector, JHEP 05 (2014) 071 [arXiv: 1403.5294] [INSPIRE].

[64] CMS collaboration, Searches for electroweak production of charginos, neutralinos and sleptons decaying to leptons and $W, Z$ and Higgs bosons in pp collisions at $8 \mathrm{TeV}$, Eur. Phys. J. C 74 (2014) 3036 [arXiv:1405.7570] [InSPIRE].

[65] M. Cacciari, G.P. Salam and G. Soyez, The anti-kt jet clustering algorithm, JHEP 04 (2008) 063 [arXiv:0802.1189] [INSPIRE].

[66] M. Cacciari and G.P. Salam, Dispelling the $N^{3}$ myth for the $k_{t}$ jet-finder, Phys. Lett. B 641 (2006) 57 [hep-ph/0512210] [INSPIRE].

[67] M. Cacciari, G.P. Salam and G. Soyez, FastJet user manual, Eur. Phys. J. C 72 (2012) 1896 [arXiv:1111.6097] [INSPIRE].

[68] R. Gavin and M.K. Trenkel, SUSY QCD corrections to electroweak gauge boson production with an associated jet at the LHC, JHEP 01 (2012) 036 [arXiv:1109.3445] [INSPIRE].

[69] M.R. Buckley, J.D. Lykken, C. Rogan and M. Spiropulu, Super-razor and searches for sleptons and charginos at the LHC, Phys. Rev. D 89 (2014) 055020 [arXiv: 1310.4827] [INSPIRE].

[70] T. Melia, P. Nason, R. Rontsch and G. Zanderighi, $W^{+} W^{-}, W Z$ and $Z Z$ production in the POWHEG BOX, JHEP 11 (2011) 078 [arXiv:1107.5051] [INSPIRE].

[71] J.F. Gunion and S. Mrenna, A study of SUSY signatures at the Tevatron in models with near mass degeneracy of the lightest chargino and neutralino, Phys. Rev. D 62 (2000) 015002 [hep-ph/9906270] [INSPIRE].

[72] C. Han et al., Probing light Higgsinos in natural SUSY from monojet signals at the LHC, JHEP 02 (2014) 049 [arXiv: 1310.4274] [INSPIRE].

[73] P. Schwaller and J. Zurita, Compressed electroweakino spectra at the LHC, JHEP 03 (2014) 060 [arXiv:1312.7350] [INSPIRE].

[74] H. Baer, A. Mustafayev and X. Tata, Monojets and mono-photons from light higgsino pair production at LHC14, Phys. Rev. D 89 (2014) 055007 [arXiv: 1401.1162] [INSPIRE]. 